\title{
The role of sympatho-inhibition in combination treatment of obesity-related hypertension
}

\author{
Revathy Carnagarin ${ }^{1}$, Cynthia Gregory ${ }^{1}$, Omar Azzam ${ }^{1}$, Graham S Hillis ${ }^{1,2}$, Carl Schultz ${ }^{1,2}$, \\ Gerald F Watts ${ }^{1,2}$, Damon Bell ${ }^{1,2}$, Vance Matthews ${ }^{1}$, and Markus P Schlaich ${ }^{1,2,3}$ \\ ${ }^{1}$ Dobney Hypertension Centre, School of Medicine - Royal Perth Hospital Unit, University \\ of Western Australia, Perth, and ${ }^{2}$ Department of Cardiology and ${ }^{3}$ Department of \\ Nephrology, Royal Perth Hospital, Perth, Australia.
}

Word count: 2902 words (excluding references)

\section{Correspondence to:}

Professor Markus Schlaich

Dobney Chair in Clinical Research

School of Medicine - Royal Perth Hospital Unit

The University of Western Australia

Level 3, MRF Building, Rear 50 Murray St,

Perth WA 6000; Australia

Phone: +61 892240334 Fax: +61 892240374

email: markus.schlaich@uwa.edu.au 


\begin{abstract}
Obesity related hypertension is commonly characterized by increased sympathetic nerve activity and is therefore acknowledged as a predominantly neurogenic form of hypertension. The sustained sympatho-excitation not only contributes to the rise in blood pressure but elicits a vicious cycle which facilitates further weight gain and progression of associated comorbidities. While weight loss and exercise remain at the forefront of therapy for obesity and obesity related hypertension, the difficulties in achieving and maintaining long-term weight loss with life-style measures and the variable blood pressure response to weight loss often necessitate prescription of antihypertensive drug therapy. Remarkably there are no specific recommendations for pharmacologic treatment for obese patients with arterial hypertension in any of the current guidelines and general principles of antihypertensive treatment are applied. The use of $\beta$-blockers and diuretics is commonly discouraged as first or second line therapy due to their unfavourable metabolic effects. This review explores evolving therapeutic strategies which based on their interference with pathophysiologic mechanism relevant in the context of obesity may guide optimized treatment of obesity related hypertension.
\end{abstract}

Keywords: Obesity related hypertension; sympathetic activation; pharmacotherapy; antihypertensive medications; pathophysiology. 


\section{Introduction}

The obesity pandemic is reaching a staggering expansion with a current prevalence of 40$60 \%$ and an expected increase to 75\% of females and 83\% of males by 2025 in industrialized countries such as Australia and the US. Obesity along with its associated co-morbidities and mortality has emerged as a worldwide threat to population health and enormous economic burden in many countries [1]. According to the World Health Organization, 'Obesity is one of the most important contributors to ill health'. There is concern that any gains made in reducing cardiovascular disease may actually be reversed by the obesity epidemic [2]. Given the large increase in the number of overweight and obese individuals, the proportion of hypertensive patients who are obese is likely to increase sharply in the future. Thus, many patients with arterial hypertension are exposed to the additional metabolic and cardiovascular risk associated with obesity.

Obesity is more than a co-morbid condition because it can cause or worsen arterial hypertension in susceptible individuals. Large-scale epidemiological studies such as NHANES and Framingham suggest a tight correlation between body weight and blood pressure. Statistical analysis of this data suggested that $60-70 \%$ of hypertension may be directly attributable to excess adiposity, both in men and in women [3]. The age-adjusted relative risk for the development of hypertension was 1.75 in men and 1.46 in women [4]. Regression models based on random population samples, corrected for the age-related rise in blood pressure (BP) demonstrated a stable linear relationship of adiposity with BP across developed and developing countries. In this cross sectional health examination survey involving 3116 adults not treated for hypertension (age range: 35-64), the systolic BP increased by $1 \mathrm{mmHg}$ for a gain of $1.7 \mathrm{~kg} / \mathrm{m} 2$ and $4.5 \mathrm{~cm}$ (men), or $1.3 \mathrm{~kg} / \mathrm{m} 2$ and $2.5 \mathrm{~cm}$ (women) in body mass index or waist circumference, respectively [5]. Another 11 year prospective population study confirmed a strong positive causal association between changes in BMI and changes in BP [6]

Clinical trials indicated that loss of original body weight (around 10\%) resulted in clinically significant reductions in blood pressure and cardiovascular mortality [7, 8]. Contemporaneous studies indicated that increased adiposity resulted in metabolic abnormalities which in concert with hypertension, resulted in the development of cardiorenal and metabolic syndrome [9,10], features that are mediated to a large extent by sustained activation of the sympathetic nervous system [11]. Further support for a dominant role of the sympathetic nervous system in this context stems from studies demonstrating sympathoinhibitory effects of diet and/or exercise-induced weight loss on BP and relevant metabolic 
markers [12]. Thus the concept of sympathetic overdrive has emerged as a major cause of obesity-induced morbidity and cardiovascular mortality and functions in a feedforward manner to sustain obesity itself.

While lifestyle modification is clearly effective in reducing excess body weight and improving various aspects of the cardiovascular (CV) and metabolic consequences of obesity, the majority of patients fail to implement and sustain appropriate lifestyle changes. Antihypertensive combination therapy is very frequently required in obesity-related hypertension $(\mathrm{OH})$ for $\mathrm{CV}$ risk reduction and BP lowering. While current hypertension guidelines do not provide specific recommendations for treatment of $\mathrm{OH}$, inhibitors of the renin-angiotensin system such as ACE inhibitors (ACEI) or angiotensin receptor blockers (ARBs) are widely considered as first choice due to their favourable side effect profile, documented cardiorenal protection and additional beneficial metabolic effects. Most hypertension guidelines generally recommend calcium channel blockers (CCBs) and diuretics as the appropriate add-on drugs if combination therapy is required. In the context of $\mathrm{OH}$, CCBs appear as a preferred combination due to their powerful BP lowering capacity, particularly in combination with RAS blockers and their neutral effect on metabolic parameters, whereas diuretics seem less favourable in view of their unwanted metabolic side effects including worsening of insulin resistance and exacerbation of gout. Given the important role of sympathetic activation in obesity the use of $\beta$-blockers may seem as plausible antihypertensive option, however their association with weight gain and other adverse metabolic consequences such as worsening of insulin resistance makes them less than ideal in $\mathrm{OH}$. Robust evidence for a potentially preferable antihypertensive combination therapy for $\mathrm{OH}$ has yet to be established. Here we review important aspects including the pathophysiology underlying $\mathrm{OH}$, available evidence for existing management strategies and other relevant findings that may guide the identification of a preferred combination treatment tailored to the specific features and complications of $\mathrm{OH}$.

\section{Pathophysiologyof Obesity Hypertension}

$\mathrm{OH}$ is a complex disorder resulting from the interplay of multiple factors perturbing normal cardiometabolic homeostasis (Figure 1). In lean hypertensive individuals, much of the hypertension is driven by an increase in systemic vascular resistance. By contrast, in obese hypertensive individuals, the hypertension is at least partly explained by increased cardiac 
output [13]. The increase in cardiac output may result from sympathetic activation and plasma volume expansion due to sodium retention [14]. Obese hypertensive patients clearly exhibit an increase in sympathetic activity as assessed by noradrenaline spillover, particularly sympathetic outflow to the renal vascular bed further promoting volume retention, alterations in renal blood flow, and release of renin from the juxtaglomerular apparatus, thereby engaging the renin-angiotensin-aldosterone cascade [15]. Baroreflex dysfunction and the sleep apnea syndrome, a common co-morbidity in obese subjects, may also contribute to sympathetic overactivity [16, 17]. Pharmacological studies and direct recordings of sympathetic nerve activity suggest that the sympathetic nervous system contributes substantially to obesity-associated arterial hypertension [18, 19] and is characterized by distinct differences in the firing pattern of sympathetic neurons in human obesity [20].

Sympathetic tone is also exaggerated by the obesity induced derangement in pressure natriuresis. As stated by Guyton's hypothesis, sustained hypertension is the result of abnormal relationship between arterial pressure and natriuresis. Whereas increased pressure results in enhanced sodium excretion to reduce hypertension in the normal scenario [21], the obesity-related alterations such as the enhanced sympathetic tone, activation of the reninangiotensin-aldosterone system (RAAS), hyperinsulinemia, and renal structural changes significantly impair the pressure-natriuresis curve. Sympathetic blockade (combined $\alpha$ - and $\beta$-blockade) prevents $\mathrm{OH}$ in experimental animals and in patients [18, 22-25]. Renal denervation altered the pressure-natriuresis relationship in $\mathrm{OH}$ animal models [22-29]. Similarly, leptin secreted by adipocytes to produce satiety and weight loss increases sympathetic overdrive to induce thermogenesis, at least in experimental studies. Also, hyperleptinemia stimulates the hypothalamic pro-opiomelanocortin (an important weight modulator) pathway and further exaggerates central sympathetic outflow. Mutations in the melanocortin 4 receptor produce hypertension in man, thereby demonstrating the regulatory capacity of the melanocortin pathway in blood pressure and weight regulation [24, 30].

Exaggerated RAAS activation with high circulating renin, angiotensinogen, and angiotensin II, despite augmented renal sodium retention is seen in $\mathrm{OH}$ [23-24, 27, 29, 31]. Adipose tissue endogenously synthesizes and increases the circulating angiotensinogen in obesity which enhances the production of angiotensin I and angiotensin II [31]. Angiotensin II further increases renal tubular sodium reabsorption and stimulates synthesis of aldosterone, thereby promoting sodium and fluid retention. Similarly, obesity associated 
hyperinsulinenmia [27, 28] elevates arterial pressure as insulin enhances tubular sodium reabsorption. The effect of angiotensin-converting enzyme inhibitors and peroxisome proliferator-activated receptor gamma agonists on blood pressure supports the role of the RAAS and hyperinsulinemia in this context [32-34]. The endocannabinoid system, though less well-understood may have a role in $\mathrm{OH}$. Obesity is associated with increased levels of endocannabinoids in several tissues and in the circulation. The cannabinoid receptor 1 inverse agonists, rimonabant and taranabant produced weight loss and ameliorated obesity-related metabolic impairments [32-34].

\section{Strategies for the management of Obesity Hypertension}

Obesity and arterial hypertension frequently coexist. Obesity can cause or worsen arterial hypertension and is a common cause of resistance to antihypertensive treatment. Moreover, the associated risk profile differs markedly between lean and obese patients with arterial hypertension. Current guidelines do not provide specific recommendations for pharmacologic treatment of obese patients with arterial hypertension. This state of affairs exists due to the lack of data from clinical trials, an incomplete understanding of the underlying pathophysiology, and perhaps neglect by the scientific and clinical community. The various management strategies for OHT are summarised in Figure 2.

\section{Lifestyle modification}

Weight loss and exercise remain at the forefront of therapy for obesity and OH. Short term exercise training has consistently been shown to be associated with a reduction in sympathetic activity and an improvement in blood pressure. We have shown that in subjects with the metabolic syndrome, weight loss following a 12 week dietary program was accompanied by a significant reduction in sympathetic nervous activity and an improvement in all components of the metabolic syndrome [13, 35]. In clinical practice however, the majority of patients fail to implement and sustain appropriate lifestyle changes in the long term.

\section{Pharmacologic antihypertensive treatment}

Given the difficulties in achieving and maintaining long-term weight loss with life-style measures and the variable blood pressure response to weight loss, many obese hypertensive patients ultimately require antihypertensive combination drug therapy, with 2 or 3 antihypertensive drugs to achieve BP control [36]. Remarkably, current hypertension 
guidelines do not provide specific recommendations for the choice of antihypertensive medications. Indeed, there are no larger trials addressing this issue [37]. Therapeutic decisions have to be made on the basis of few existing data, including our current understanding of the mechanisms involved in $\mathrm{OH}$ and the presence or absence of target organ damage or cardiovascular disease in individual patients. Furthermore, attention to potential beneficial effects beyond blood pressure lowering or adverse and unwanted metabolic effects of certain drug classes may favour or limit their respective use. For example, $\beta$-blockers reduce cardiac output and renin activity, both of which have been shown to be increased in obese patients. It is therefore not surprising that $\beta$-blockers alone [38], or in combination with $\alpha$-adrenoreceptor blockers [39], were more effective in decreasing blood pressure in obese than in lean hypertensive individuals. However, substantial limitations for the use of $\beta$ blockers, especially in young obese hypertensive patients without cardiac and renal complications, are related to their well described unfavourable effects on glucose metabolism and increase in body weight $[40,41]$. Whether newer vasodilating $\beta$-blockers may be better alternatives remains to be determined. Similarly, while diuretics are useful in counteracting the volume overload frequently evident in obesity hypertension, their adverse metabolic effects and their capacity to further exaggerate the already increased sympathetic drive renders them a suboptimal choice.

In view of their broad spectrum of beneficial effects, excellent tolerability, proven cardiovascular protection and beneficial effects on metabolic aspects, inhibitors of the RAAS, in particular ACE-inhibitors and ARBs are widely considered to be the most appropriate first line drug for antihypertensive treatment of obese patients [42].

CCBs are effective antihypertensive drugs and considered neutral in regards to their metabolic effect. However, some data suggest that CCBs may be less effective in severely obese subjects, possibly through their accompanying sympathoexcitation. Furthermore, fluid retention and peripheral oedema can limit their utility [43].

Given the unequivocal evidence for an important role of increased sympathetic outflow in many of the features characteristic of $\mathrm{OH}$, it is perhaps surprising that centrally acting sympatholytic agents such as Imidazoline I1 agonists, which effectively inhibit sympathetic outflow at the level of the rostral ventrolateral medulla (Figure 1), are not frequently 
considered in the treatment of $\mathrm{OH}$. Aside from their blood pressure lowering capacity which is comparable to that of other drug classes, agents such as moxonidine have been demonstrated to improve glucose metabolism, insulin sensitivity and dyslipidemia [44, 45]. Furthermore, their use has been associated with a reduction in the progression of microalbuminuria and renal dysfunction, common features observed in obese subjects [46, 47]. Other beneficial effects on end organ function include a reduction in left ventricular hypertrophy [48] and improvement in endothelial function [49].

Most recently, another potentially highly relevant link in the current context has been described between sympathetic activation and expression of the sodium glucose cotranporter-2 (SGLT-2) [50]. SGLT-2 is relevant for renal sodium handling and inhibitors of SGLT-2 have shown to improve glucose control but perhaps more importantly to reduce CV events in patients with diabetes [51]. In this study by Matthews et al. noradrenaline, the main neurotransmitter of the sympathetic nervous system was shown to upregulate SGLT-2 expression in human proximal tubular cells. [50]. Inhibition of central sympathetic outflow with the resulting reduction in circulating noradrenaline could be an important mediator of sympathoinhibition-induced improvement of glucose metabolism via SGLT-2 regulation, thereby potentially further favouring such an approach in obesity hypertension.

A recently published large observational study in 5603 patients with overweight/obesity related BP elevation and metabolic disturbances (MERSY study) investigating the effects of moxonidine alone or in combination with other BP lowering medications demonstrated favourable effects not only on BP but a number of relevant metabolic markers in a primary care setting [52]. While clearly limited by the observational nature and the lack of a control group, the findings from this study provide evidence for a substantial BP lowering effect and clinically relevant improvements in metabolic markers such as body weight, fasting glucose levels and lipid profile.

In addition, a small, randomized, open parallel study in obese hypertensive subjects compared the effects of moxonidine $(0.2 \mathrm{mg}-0.4 \mathrm{mg} / \mathrm{d} ; \mathrm{n}=19, \mathrm{BMI}=35.4 \pm 1.7 \mathrm{~kg} / \mathrm{m} 2)$ and amlodipine $(5-10 \mathrm{mg} / \mathrm{d} ; \mathrm{n}=21 ; \mathrm{BMI}=35.9 \pm 1.4 \mathrm{~kg} / \mathrm{m} 2)$ as stand-alone therapy [53]. The reduction in ambulatory systolic BP was substantial in both groups (moxonidine: from $144.1 \pm 4.2$ to $133.8 \pm 3.1 \mathrm{mmHg}$ vs amlodipine: from $145.4 \pm 4.4$ to $129.0 \pm 1.9 \mathrm{mmHg}$ ) without a statistically significant difference in the change in BP between the two groups. However, up- 
titration of medication was to the highest recommended dose of amlodipine $(10 \mathrm{mg} / \mathrm{d})$ but only to a moderate dose of moxonidine $(0.4 \mathrm{mg} / \mathrm{d}$, highest recommended dose is $0.6 \mathrm{mg} / \mathrm{d}$ ) [54]. Nevertheless, moxonidine administration reduced the supine arterial plasma levels of adrenaline from $63.2 \pm 6.6$ to $49.0 \pm 6.7 \mathrm{pg} / \mathrm{ml}(\mathrm{p}<0.005)$, the supine arterial plasma levels of noradrenaline from $187.9 \pm 10.7$ to $149.7 \pm 13.2 \mathrm{pg} / \mathrm{ml}(\mathrm{p}<0.01)$ and the orthostatic venous plasma levels of noradrenaline from $258.6 \pm 25.0$ to $190.3 \pm 16.4 \mathrm{pg} / \mathrm{ml}$ ( $\mathrm{p}=0.03$ ). None of those variables were changed by amlodipine. The plasma levels of leptin and insulin $120 \mathrm{~min}$ after a glucose load decreased after moxonidine administration from $27.2 \pm 3.5$ to $22.6 \pm 2.9$ $\mathrm{pg} / \mathrm{ml}(\mathrm{p}<0.05)$ and from $139.7 \pm 31.2$ to $76.0 \pm 15.2 \mathrm{U} / \mathrm{ml}(\mathrm{p}<0.05)$, respectively. Amlodipine, however, did not modify those variables. While limited by its small sample size, this study provides important preliminary data to support the pathophysiologically sound notion that in obesity hypertension a therapeutic approach targeting central sympathetic outflow may not only provide substantial and effective BP lowering but beneficially impact on a number of relevant metabolic markers relevant for cardiovascular risk. A larger scale active comparator study, ideally on background medication with RAS blockade to properly compare the BP and metabolic benefits of a CCB vs Imidazoline I agonist would be highly relevant to inform on the most adequate antihypertensive approach for obesity hypertension when combination therapy is warranted.

\section{Conclusions and future directions}

Antihypertensive combination treatment is required in the vast majority of hypertensive patients, particularly in obese hypertensives. Inhibitors of the renin angiotensin system are considered first line choice due to additional beneficial effects on metabolic control. Most hypertension guidelines generally recommend CCBs and diuretics as preferred combination partners. However, in obesity related hypertension diuretics have unwanted metabolic side effects, leaving CCBs as a preferred second line choice. We and others have provided substantial evidence to indicate that sympathetic overactivity is a cardinal feature of obesity related hypertension that not only impacts on BP but also metabolic control. Targeting sympathetic overactivity therapeutically may therefore be of specific use in $\mathrm{OH}$ and combining RAS blockade with a centrally acting sympatholytic agent compared to a CCB (amlodipine) may provide better blood pressure and metabolic control and have positive implications for cardiovascular risk reduction. Sympathoinhibition may therefore emerge as a preferred second line treatment choice for obesity related hypertension, a proposition that requires thorough clinical testing. 


\section{Sources of Funding}

None

\section{Disclosures}

MPS is supported by an NHMRC Research Fellowship and has received consulting fees, and/or research support from Abbott. None of the other authors declare any conflict of interest relevant to this manuscript. 


\section{References}

Papers of particular interest, published recently, have been highlighted as: • Of importance

1. Alwan A. World Health Organization. Global Status Report on Non-communicable Diseases 2010. 2010.

2. WHO. Obesity - preventing and managing the global epidemic. WHO Consultation on Obesity, Geneva, 3-5 June 1997. Geneva: WHO; 1997.

3. Must A et al. The disease burden associated with overweight and obesity. JAMA 1999; 282: 1523-1529.

4. Wilson PW et al. Overweight and obesity as determinants of cardiovascular risk: the Framingham experience. Arch Intern Med 2002; 162: 1867-1872.

5. Doll S et al. Body mass index, abdominal adiposity and blood pressure: consistency of their association across developing and developed countries. Int J Obes 2002; 26: 4857.

6. Droyvold WB et al. Change in body mass index and its impact on blood pressure: a prospective population study. Int J Obes 2005; 29: 650-655.

7. Neter JE et al. Influence of weight reduction on blood pressure: a meta-analysis of randomized controlled trials. Hypertension 2003; 42,878-884.

8. Appel LJ et al. Dietary approaches to prevent and treat hypertension: a scientific statement from the American Heart Association. Hypertension 2006; 47, 296-308.

9. Alberti KG \& Zimmet PZ. Definition, diagnosis and classification of diabetes mellitus and its complications. Part 1: diagnosis and classification of diabetes mellitus provisional report of a WHO consultation. Diabet Med 1998; 15: 539-553.

10. Alberti KG et al. Harmonizing the metabolic syndrome. A joint interim statement of the International Diabetes Federation Task Force on Epidemiology and Prevention; National Heart, Lung, and Blood Institute; American Heart Association; World Heart Federation; International Atherosclerosis Society; and International Association for the Study of Obesity. Circulation 2009; 120: 1640-1645. 
11. Schlaich $\mathrm{M}$ et al. Metabolic syndrome: a sympathetic disease? The lancet Diabetes \& endocrinology. 2015; 3: 148-157.

12. Straznicky NE et al. Effects of dietary weight loss on sympathetic activity and cardiac risk factors associated with the metabolic syndrome. J Clin Endocrinol Metab. 2005; 90: 5998-6005.

13. Stelfox HT et al. Hemodynamic monitoring in obese patients: the impact of body mass index on cardiac output and stroke volume. Crit Care Med 2006; 34:1243-1246.

14. Strazzullo P et al. Abnormalities of renal sodium handling in the metabolic syndrome. Results of the Olivetti Heart Study. J Hypertens 2006; 24: 1633-1639.

15. Rumantir MS et al. Neural mechanisms in human obesity-related hypertension. J Hypertens 1999; 17: 1125-1133.

16. Grassi $\mathrm{G}$ et al. Adrenergic and reflex abnormalities in obesity-related hypertension. Hypertension 2000; 36: 538-542.

17. Narkiewicz K et al. Sympathetic activity in obese subjects with and without obstructive sleep apnea. Circulation 1998; 98: 772-776.

18. Wofford MR et al. Antihypertensive effect of alpha- and beta-adrenergic blockade in obese and lean hypertensive subjects. Am J Hypertens 2001; 14: 694-698.

19. Shibao C et al. Autonomic contribution to blood pressure and metabolism in obesity. Hypertension 2007; 49: 27-33.

20. Lambert E et al. Differing pattern of sympathoexcitation in normal-weight and obesityrelated hypertension. Hypertension 2007; 50: 862-868.

21. WHO. Obesity and Overweight: Fact Sheet number 311. 2011 [updated 2011; cited]; Available from: http://www.who. int/mediacentre/factsheets/fs311/en/index.html (accessed 8 November 2009).

22. Frohlich ED. Clinical management of the obese hypertensive patient. Cardiol Rev. 2002; 10: 127-138.

23. HallJE. Pathophysiology of obesity hypertension. Curr Hypertens Rep. 2000; 2: 139147.

24. Hall JE et al. Mechanisms of obesity-associated cardiovascular and renal disease. Am J Med Sci. 2002; 324:127-137.

25. Wofford MR, Hall JE. Pathophysiology and treatment of obesity hypertension. Curr Pharm Des. 2004; 10: 3621-3637.

26. Victor RG, Shafiq MM. Sympathetic neural mechanisms in human hypertension. Curr Hypertens Rep. 2008; 10: 241-247. 
27. Sarzani R et al. Renin-angiotensin system, natriuretic peptides, obesity, metabolic syndrome, and hypertension: an integrated view in humans. J Hypertens. 2008; 26: 831-843.

28. Bloomgarden ZT. Obesity, hypertension, and insulin resistance. Diabetes Care 2002; 25: 2088-2097.

29. Re RN. The clinical implication of tissue renin angiotensin systems. Curr Opin Cardiol. 2001; 16: 317-327.

30. Greenfield JR et al. Modulation of blood pressure by central melanocortinergic pathways. N Engl J Med. 2009; 360: 44-52.

31. Dzau VJ \& Re R. Tissue angiotensin system in cardiovascular medicine: a paradigm shift? Circulation. 1994; 89: 493-498.

32. Engeli S. Dysregulation of the endocannabinoid system in obesity. J Neuroendocrinol. 2008; 20: 110-115.

33. Sarzani R. Endocannabinoids, blood pressure and the human heart. J Neuroendocrinol. 2008; 20 :58-62.

34. Grassi $G$ et al. Blood pressure lowering effects of rimonabant in obesity-related hypertension. J Neuroendocrinol. 2008; 20: 63-68.

35. - Straznicky NE et al. The effects of weight loss versus weight loss maintenance on sympathetic nervous system activity and metabolic syndrome components. J Clin Endocrinol Metab. 2011; 96: E503-E508. A prospective lifestyle intervention trial that identified divergent effects of successful weight loss maintenance on wholebody norepinephrine spillover rate and MSNA suggesting organ-specific differentiation in SNS adaptation to weight loss under conditions of negative vs. stable energy balance.

36. Uretsky $\mathrm{S}$ et al. Obesity paradox in patients with hypertension and coronary artery disease. Am J Med. 2007; 120: 863-870.

37. Dentali $\mathrm{F}$ et al. Management of hypertension in overweight and obese patients: a practical guide for clinicians. Curr Hypertens Rep. 2005; 7: 330-336.

38. Schmieder RE et al. Obesity as a determinant for response to antihypertensive treatment. Br Med J 1993; 307: 537-540.

39. Wofford MR et al. Antihypertensive effect of alpha- and beta-adrenergic blockade in obese and lean hypertensive subjects. Am J Hypertens 2001; 14: 694-698. 
40. Gress TW et al. Hypertension and antihypertensive therapy as risk factors for type 2 diabetes mellitus. Atherosclerosis Risk in Communities Study. N Engl J Med 2000; 342: 905-912.

41. Sharma AM et al. Hypothesis: beta-adrenergic receptor blockers and weight gain: a systematic analysis. Hypertension 2001; 37: 250-254.

42. Jordan $\mathrm{J}$ et al. Hemodynamic and metabolic responses to valsartan and atenolol in obese hypertensive patients. J Hypertens 2005; 23: 2313-2318.

43. Jordan $\mathrm{J}$ et al. Antihypertensive treatment in patients with class 3 obesity. Ther Adv Endocrinol Metab 2012; 3: 93-98.

44. Chazova I et al. Moxonidine improves glycaemic control in mildly hypertensive, overweight patients: a comparison with metformin. Diabetes Obes Metab. 2006; 8: 45665.

45. Haenni A \& Lithell H. Moxonidine improves insulin sensitivity in insulin-resistant hypertensives. J Hypertens. 1999; 17: S29-S35.

46. Strojek $\mathrm{K}$ et al. Lowering of microalbuminuria in diabetic patients by a sympathicoplegic agent: novel approach to prevent progression of diabetic nephropathy? J Am Soc Nephrol. 2001;12: 602-605.

47. Vonend $\mathrm{O}$ et al. Moxonidine treatment of hypertensive patients with advanced renal failure. J Hypertens. 2003; 21: 1709-1717.

48. Prichard $\mathrm{BN}$ et al. Moxonidine: a new antiadrenergic antihypertensive agent. J Hypertens. 1999; 17: S41 -S54.

49. Topal E et al. The effect of moxonidine on endothelial dysfunction in metabolic syndrome. Am J Cardiovasc Drugs 2006; 6: 343-348.

50. - Matthews VB et al. Role of the sympathetic nervous system in regulation of the sodium glucose cotransporter 2. J Hypertens. 2017; 35: 2059-2068. This is the first study to identify the importance of SNS-SGLT2 cross talk that accounts for SNSinduced alterations in glucose metabolism and SGLT2 inhibition with dapagliflozin resulted in cardiovascular and renal protection.

51. - Zinman B et al. EMPA-REG OUTCOME Investigators. 27 Version 2, dated 25 June 2016 Empagliflozin, Cardiovascular Outcomes, and Mortality in Type 2 Diabetes. N Engl J Med. 2015 ; 373: 2117-2128. A randomized control trial where type 2 diabetics at high risk for cardiovascular events received empagliflozin along with standard care had a lower rate of the primary composite cardiovascular outcome and death as compared with placebo. 
52. - Chazova I \& Schlaich MP. Improved Hypertension Control with the Imidazoline Agonist Moxonidine in a Multinational Metabolic Syndrome Population: Principal Results of the MERSY Study. Int J Hypertens. 2013; doi: 10.1155/2013/541689. A large, multinational study that appraised the effects of moxonidine on blood pressure, anthropometric, lipid, and metabolic parameters of the metabolic syndrome in routine clinical practice.

53. Schlaich MP et al. European Society of Hypertension Working Group on Obesity; Australian and New Zealand Obesity Society. European Society of Hypertension Working Group on Obesity Obesity-induced hypertension and target organ damage: current knowledge and future directions. J Hypertens. 2009; 27: 207-211.

54. Sanjuliani AF et al. Selective imidazoline agonist moxonidine in obese hypertensive patients. Int J ClinPract. 2006; 60: 621-629. 
Figure 1: Pathophysiologic aspects of obesity- related hypertension

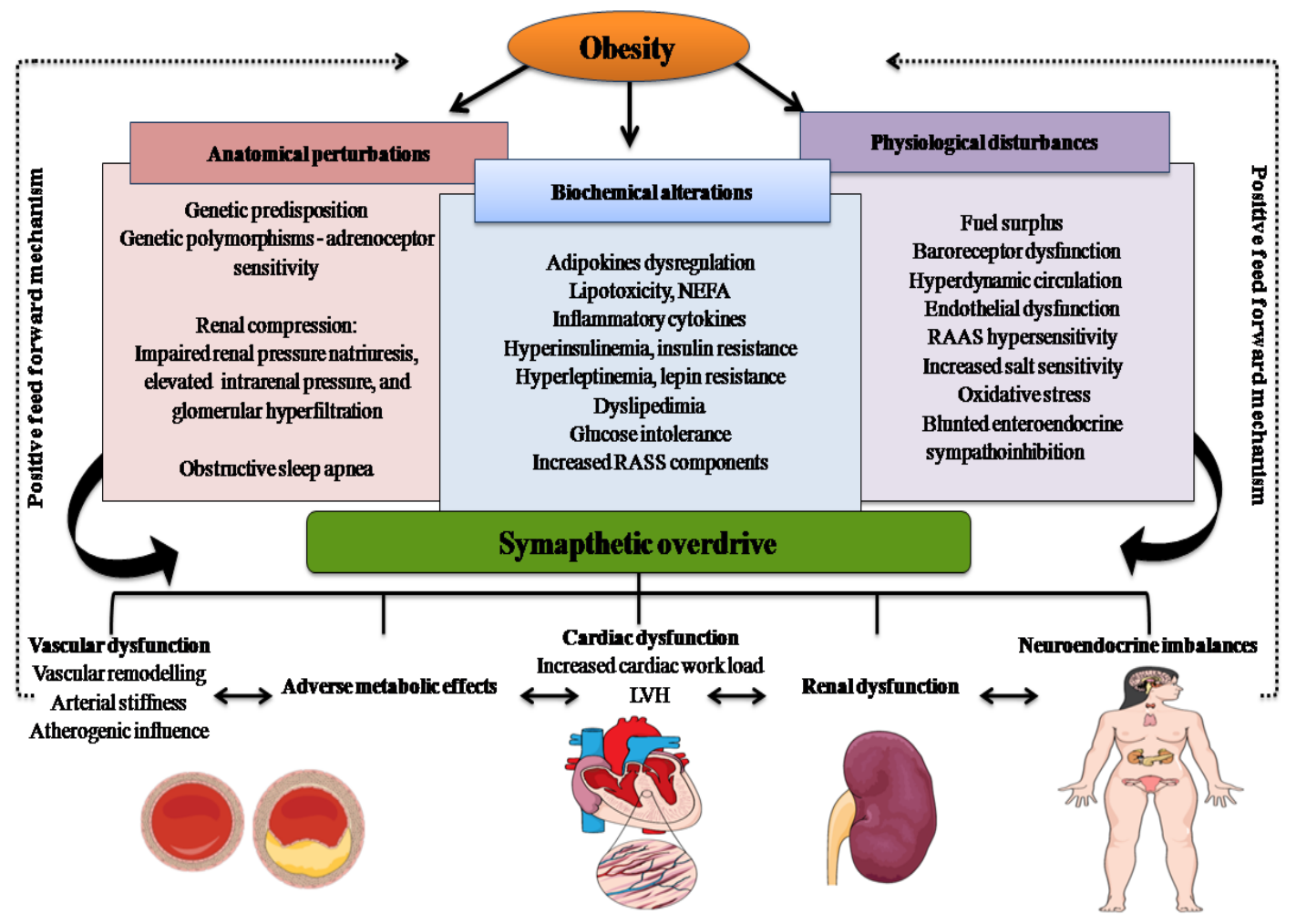


Figure 2: The various management strategies for obesity-related hypertension

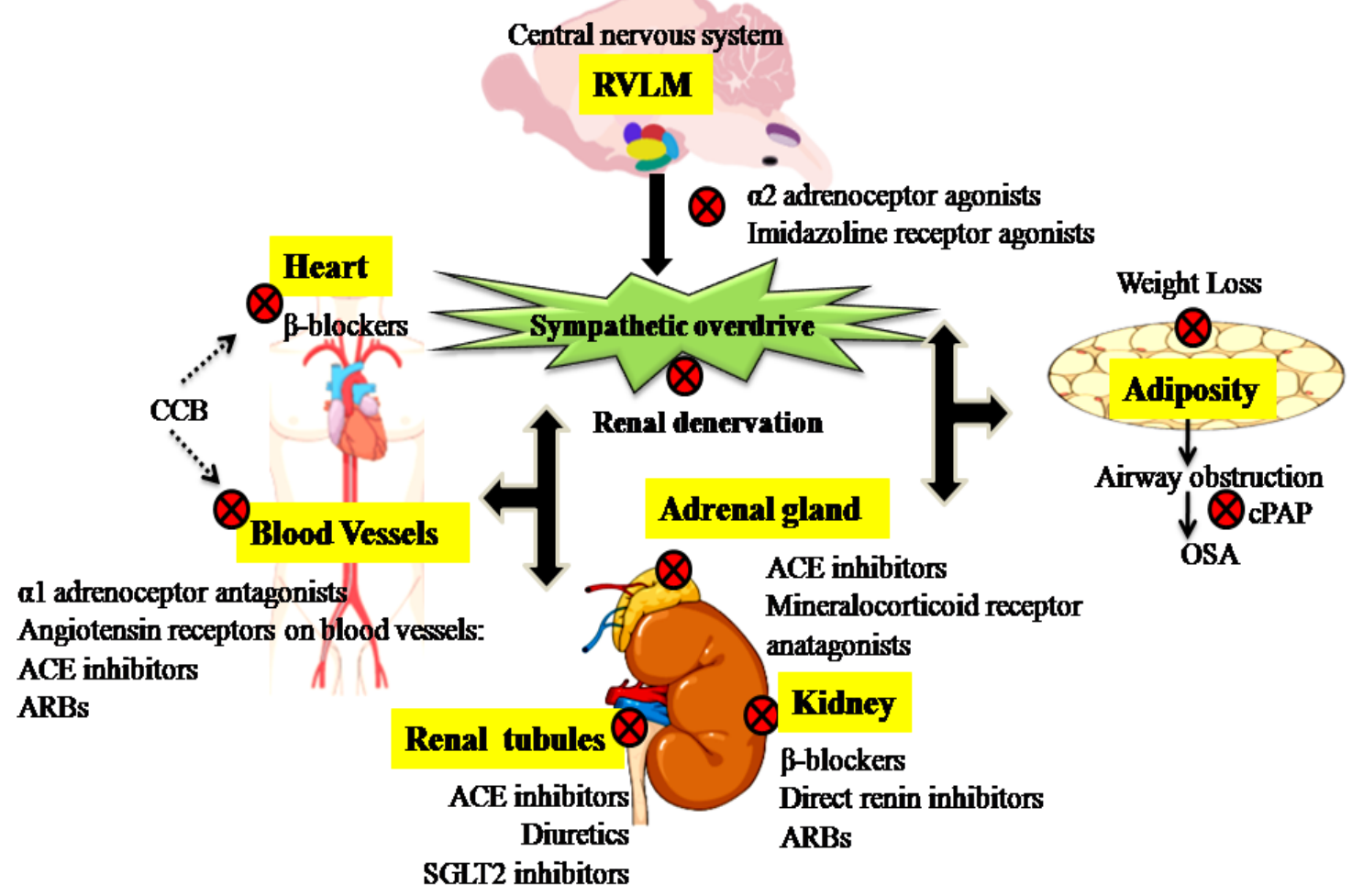


